\title{
Grade III Solitary Fibrous \\ Tumor/Hemangiopericytoma: A Rare Case of a World Health Organization Grade III Anaplastic Hemangiopericytoma
}

\author{
Matthew Jenson ${ }^{1}$, Dalys Haymes ${ }^{1}$, Jeet Patel ${ }^{1}$ \\ 1. Radiology, University of Florida College of Medicine, Jacksonville, USA \\ Corresponding author: Matthew Jenson, matthew.jenson@jax.ufl.edu
}

\begin{abstract}
Solitary fibrous tumors (SFTs) and hemangiopericytomas (HPCs) have been combined into a single designation in the most recent World Health Organization (WHO) guidelines as solitary fibrous tumor/hemangiopericytoma (SFT/HPC). These rare intracranial tumors can present as WHO grade I, II, or III tumors, with the risk of recurrence, metastasis, and mortality worsening with higher-grade tumors. We present a case of a patient with a WHO grade III SFT/HPC with an emphasis on the imaging features that help differentiate this type of tumor from meningiomas, which are much more common and can appear similar. Being able to help differentiate these tumors by their imaging appearance is important to help triage and risk-stratify patient management decisions.
\end{abstract}

Categories: Radiology, Neurosurgery, Oncology

Keywords: solitary fibrous tumor, hemangiopericytoma, anaplastic hemangiopericytoma

\section{Introduction}

In the most recent (2016) World Health Organization (WHO) Classification of the Central Nervous System, a new designation of solitary fibrous tumor/hemangiopericytoma (SFT/HPC) has been created in the mesenchymal, non-meningothelial tumor subgroup [1]. These tumors are of mesenchymal origin. As with many diseases, this reorganization was brought about by the discovery that these tumors have shared genetic origins with NAB2-STAT6 fusion [2-3]. Prior to this understanding, most literature had referred to SFTs and HPCs as distinct entities.

Review began 09/24/2020 Review ended 10/08/2020 Published 10/13/2020

\section{() Copyright 2020}

Jenson et al. This is an open access article distributed under the terms of the Creative Commons Attribution License CC-BY 4.0., which permits unrestricted use, distribution, and reproduction in any medium, provided the original author and source are credited.
Tumors within the SFT/HPC designation can be WHO grade I, II, or III depending on pathologic features. WHO grade I SFT/HPC represent tumors with high collagen content and low cellularity, and correspond to tumors previously labeled as SFTs. WHO grade II SFT/HPC represents tumors with lower collagen content and higher cellularity with a vascular pattern typical of what was previously labeled as HPCs. WHO grade III SFT/HPC represents tumors that exhibit five or more mitoses per 10 high-power fields on microscopy, corresponding to what was previously labeled as anaplastic HPCs [4].

The age-adjusted incidence rate of SFT/HPC is 3.77 per 10,000,000 people [5]. Intracranial HPC (WHO grade II or III SFT/HPC) are estimated to account for $0.4 \%$ of all primary central nervous system tumors [6]. The presentation pattern of patients with SFT/HPC is nonspecific though headache is the most commonly described symptom [7].

\section{Case Presentation}

Our patient was a 65-year-old male who had presented to an outside facility with seizures. Upon further workup, he had been found to have a "brain tumor" on outside imaging and had been subsequently discharged on anti-epileptics and steroids. He had reported gradually worsening headaches and blurred vision and sought outpatient medical attention approximately one year following the initial seizure episode. Prior outside medical imaging was unavailable, and new imaging was acquired at our institution.

The initial CT head without contrast demonstrated a dense right occipital, extra-axial mass with surrounding edema and concern for dural venous sinus involvement near the torcula (Figure 1). The mass appeared heterogeneous with areas of increased density near its base, along the region of the posterior falx. CT venogram confirmed tumoral involvement in portions of the straight sinus, torcula, superior sagittal sinus, and proximal right transverse sinus (Figure 2). 


\section{Cureus}
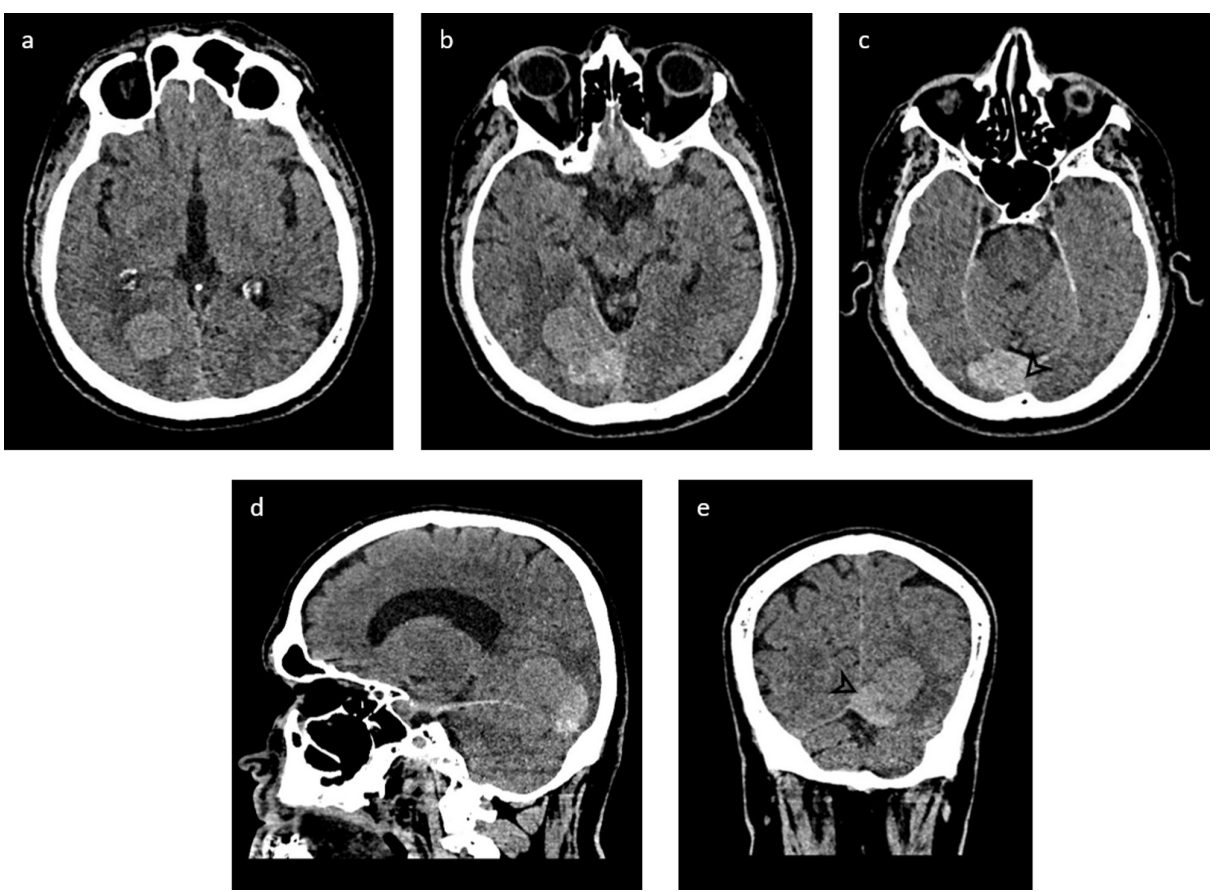

\section{FIGURE 1: Non-contrast CT head}

Axial non-contrast CT head images along the superior (a), mid (b), and inferior (c) portions of the mass followed by sagittal (d) and coronal (e) reconstructions. There is a hyperdense mass, which is seen extending from the extra-axial space near the torcula along the posterior falx. There is mild surrounding vasogenic edema in the right occipital lobe. The mass exhibits a heterogeneous appearance with a relatively more dense component seen closer to its base with suspected dural venous sinus involvement (arrowhead)

\section{CT: computed tomography}
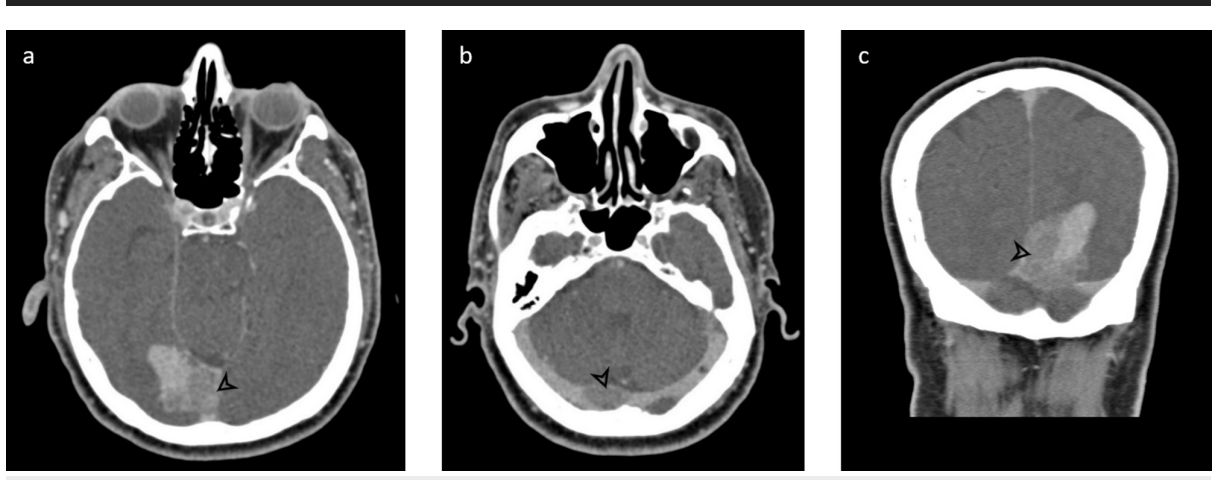

\section{FIGURE 2: CT venogram}

Post-contrast CT venogram images along the middle (a) and inferior (b) portion of the mass, as well as coronal reconstruction (c). Tumoral invasion (arrowhead) is seen within the straight sinus, most posterior aspect of the superior sagittal sinus, torcula, and proximal right transverse sinus. The remaining portions of the transverse sinuses appear well opacified

CT: computed tomography

MRI demonstrated the T1-weighted isointense mass to avidly enhance and confirmed the dural venous sinus involvement. The region at the base of the mass that exhibited increased density on the non-contrast CT was found to exhibit a T2-weighted hypointense signal and more heterogeneous enhancement. Portions of the mass demonstrated restricted diffusion, and there was some susceptibility artifact seen along the base of the mass (Figure 3). 


\section{Cureus}
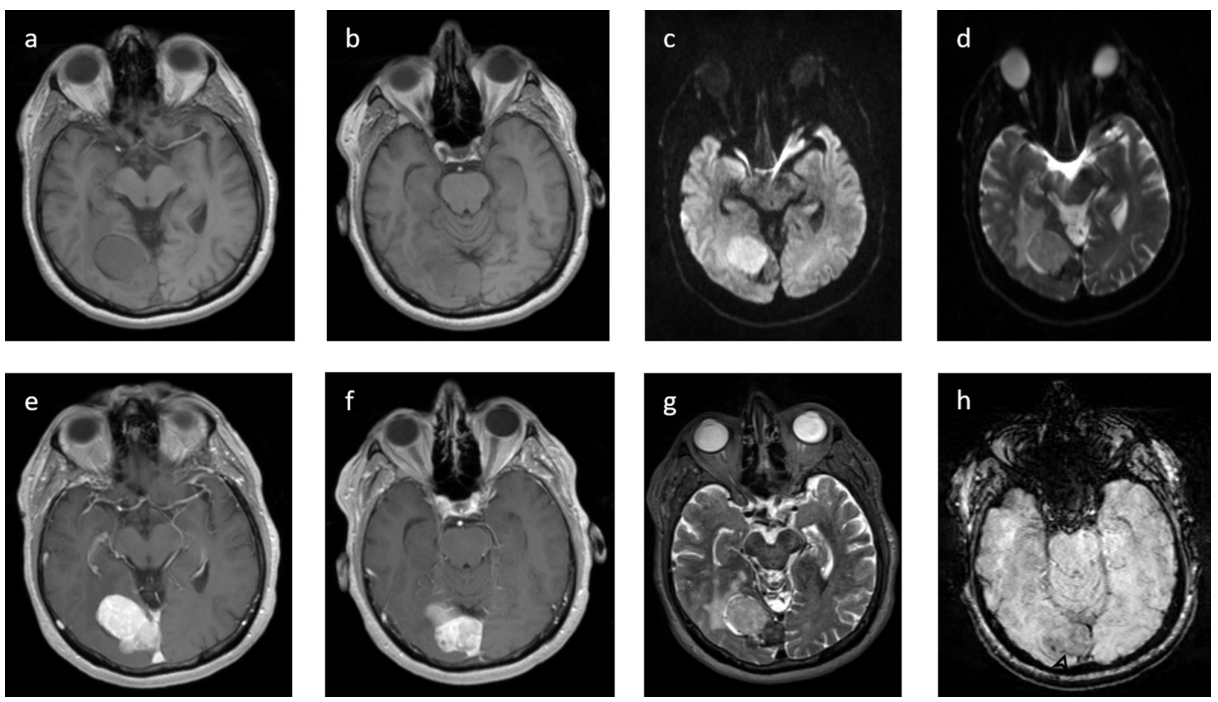

\section{FIGURE 3: MRI Brain}

T1-weighted pre-contrast images along the superior (a) and inferior (b) aspects of the mass demonstrate mostly internal isointense signal though the main, somewhat pedunculated component exhibits a slightly $\mathrm{T} 1$ weighted hypointense signal relative to the base. Diffusion (c) and ADC (d) demonstrate restricted diffusion involving the main, rounded component. T1-weighted post-contrast images along the superior (e) and inferior (f) aspects of the mass demonstrate avid enhancement, though more homogenous enhancement is seen along the superior rounded component that restricts diffusion. The T2-weighted fat-saturated image demonstrates a hypointense signal along the base of the mass with a mildly hyperintense signal along the superior, rounded component. SWI sequence demonstrates areas of susceptibility artifact (arrowhead) along the base of the mass possibly related to blood products

MRI: magnetic resonance imaging; ADC: apparent diffusion coefficient; SWI: susceptibility-weighted imaging

The patient underwent subtotal resection as portions of the mass that were invading the dural venous sinuses could not be fully resected, with a residual tumor in the dural venous sinuses demonstrated on postoperative MRI (Figure 4). The patient recovered well postoperatively and pathology results indicated WHO grade III SFT/HPC. Our institutional tumor board's recommendation was to proceed with adjuvant radiation and chemotherapy with imaging surveillance.
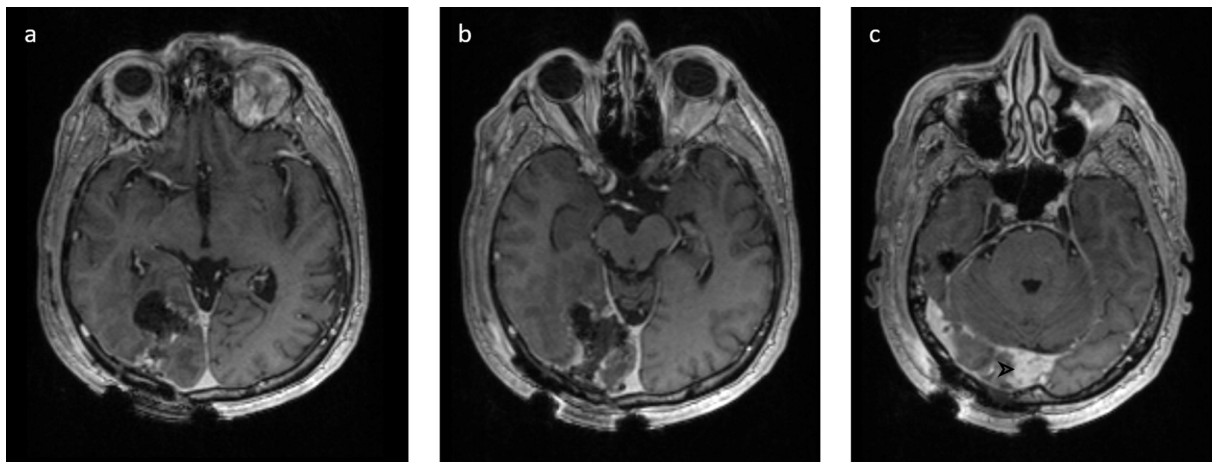

\section{FIGURE 4: Postoperative MRI}

Postoperative T1-weighted post-contrast MP-RAGE images along the super (a), mid (b), and inferior (c) aspects of the resection cavity. The majority of the resection cavity exhibits thin peripheral post-contrast enhancement, likely post-surgical in nature. However, along the inferior aspect of the resection cavity, where the tumor was previously seen to involve the straight sinus, and torcula, there is a residual thickened enhancement (arrowhead) concerning for residual tumor, corresponding to an unresected tumor on the operative report

MRI: magnetic resonance imaging; MP-RAGE: magnetization-prepared rapid gradient-echo

\section{Discussion}

Central nervous system SFTs (WHO grade I SFT/HPC) are typically thought of as benign and treated with 
surgery alone [8]. Alternatively, HPCs (WHO grade II and III SFT/HPC) are more likely to recur and metastasize extracranially and therefore more often receive radiation therapy and/or chemotherapy in addition to surgical resection [9].

SFT/HPC tumors can often share imaging characteristics with meningiomas, though meningiomas are much more common and less likely to recur or metastasize extracranially [10]. HPCs (grade II and III SFT/HPC) are usually solitary, attached to the dura, are hypervascular with avid enhancement, and can cause adjacent edema, all of which are similar imaging characteristics to that of meningiomas. However, HPCs can result in bony erosion (as opposed to the hyperostosis frequently seen in meningiomas). Intratumoral calcification is usually not a feature of HPCs in contrast to meningiomas. Additionally, a mushroom appearance of the tumor with a relatively narrow base of attachment to the dura also favors HPCs over meningiomas, which typically have a broader dural base [7].

Now that the shared genetic basis of SFTs and HPCs has come to light, some studies have sought to retrospectively see if this new classification scheme would lead to different categorization of previously treated tumors and have re-analyzed survival data. For example, in 2018, Sung et al. retrospectively reevaluated 60 patients previously diagnosed with SFT/HPC-type tumors [11]. Upon this reevaluation, there were two cases previously diagnosed as SFTs that were regraded as grade II SFT/HPC, and one case previously diagnosed as SFT that was regraded as grade III SFT/HPC. Then, using the new WHO categorization scheme, they found that the five-year survival was $100 \%$ for grade II SFT/HPC vs. $71.8 \%$ for grade III SFT/HPC.

Due to our patient initially presenting at an outside facility, it was not clear what the planned follow-up had been after the initial discharge. However, given the patient's worsening symptoms, further investigation and treatment were warranted. Understanding the different imaging features and risk of recurrence and metastasis between SFT/HPC tumors and meningiomas is important to help triage and stratify as to which cases should be more thoroughly investigated versus surveilled.

\section{Conclusions}

SFT/HPC should remain an important differential consideration when working up extra-axial masses. Despite some similarities in imaging findings, there are often clues on imaging that can help lead to including SFT/HPC as a differential consideration. As these tumors exhibit different risk of recurrence and metastasis compared to much more common meningiomas, it is important to consider the possibility of an SFT/HPC in patients.

\section{Additional Information \\ Disclosures}

Human subjects: Consent was obtained by all participants in this study. Conflicts of interest: In compliance with the ICMJE uniform disclosure form, all authors declare the following: Payment/services info: All authors have declared that no financial support was received from any organization for the submitted work. Financial relationships: All authors have declared that they have no financial relationships at present or within the previous three years with any organizations that might have an interest in the submitted work. Other relationships: All authors have declared that there are no other relationships or activities that could appear to have influenced the submitted work.

\section{References}

1. Louis DN, Perry A, Reifenberger G, et al.: The 2016 World Health Organization classification of tumors of the central nervous system: a summary. Acta Neuropathol. 2016, 131:803-20. 10.1007/s00401-016-1545-1

2. Chmielecki J, Crago AM, Rosenberg M, et al.: Whole-exome sequencing identifies a recurrent NAB2-STAT6 fusion in solitary fibrous tumors. Nat Genet. 2013, 45:131-2. 10.1038/ng.2522

3. Robinson DR, Wu YM, Kalyana-Sundaram S, et al.: Identification of recurrent NAB2-STAT6 gene fusions in solitary fibrous tumor by integrative sequencing. Nat Genet. 2013, 45:180-5. 10.1038/ng.2509

4. Bouvier C, Métellus P, de Paula AM, et al.: Solitary fibrous tumors and hemangiopericytomas of the meninges: overlapping pathological features and common prognostic factors suggest the same spectrum of tumors. Brain Pathol. 2012, 22:511-21. 10.1111/j.1750-3639.2011.00552.x

5. Kinslow CJ, Bruce SS, Rae AI, et al.: Solitary-fibrous tumor/hemangiopericytoma of the central nervous system: a population-based study. J Neurooncol. 2018, 138:173-82. 10.1007/s11060-018-2787-7

6. Rutkowski MJ, Jian BJ, Bloch O, et al.: Intracranial hemangiopericytoma: clinical experience and treatment considerations in a modern series of 40 adult patients. Cancer. 2012, 118:1628-36. 10.1002/cncr.26411

7. Smith AB, Horkanyne-Szakaly I, Schroeder JW, Rushing EJ: From the radiologic pathology archives: mass lesions of the dura: beyond meningioma-radiologic-pathologic correlation. Radiographics. 2014, 34:295312. 10.1148/rg.342130075

8. Bisceglia M, Galliani C, Giannatempo G, et al.: Solitary fibrous tumor of the central nervous system: a 15year literature survey of 220 cases (August 1996-July 2011). Adv Anat Pathol. 2011, 18:356-92. 10.1097/PAP.0b013e318229c004

9. Schiariti M, Goetz P, El-Maghraby H, Tailor J, Kitchen N: Hemangiopericytoma: long-term outcome revisited. Clinical article. J Neurosurg. 2011, 114:747-55. 10.3171/2010.6.JNS091660 


\section{Cureus}

10. Dalle Ore CL, Magill ST, Yen AJ, et al.: Meningioma metastases: incidence and proposed screening paradigm (Epub ahead of print). J Neurosurg. 2019, 1-9. 10.3171/2019.1.JNS181771

11. Sung KS, Moon JH, Kim EH, et al.: Solitary fibrous tumor/hemangiopericytoma: treatment results based on the 2016 WHO classification (Epub ahead of print). J Neurosurg. 2018, 1-8. 10.3171/2017.9.JNS171057 